\title{
LA EXPERIMENTACIÓN EN CIENCIAS NATURALES COMO ESTRATEGIA DE ALFABETIZACIÓN CIENTÍFICA
}

\section{EXPERIMENTATION IN NATURAL SCIENCES AS A SCIENTIFIC LITERACY STRATEGY}

\section{Juan Carlos Roberto Neira Morales*}

Rec.: 24-09-2020. Acept.: 12-05-2021. Publ.: 30-06-2021

DOI: http://doi.org/10.29035/ucmaule.60.102

\section{RESUMEN}

El objetivo de este estudio se centró en realizar una revisión y discusión del estado actual de la realización de las actividades experimentales como una estrategia para el logro de la alfabetización científica en etapas escolares. Actualmente, la actividad experimental más que estar asociada a actividades de resolución de problemas, está orientada a ser complemento práctico de clases teóricas, lo cual conlleva a que los estudiantes pierdan el interés por el estudio de las ciencias. Las actividades experimentales son una instancia valiosa para acercar al estudiantado al estudio de las ciencias naturales y pensamos que mantener el carácter indagatorio es fundamental para despertar el interés y lograr un adecuado desarrollo de la alfabetización científica y, por ende, pensamiento crítico.

Palabras clave: Actividad prácticas de laboratorio; alfabetización científica; pensamiento crítico; enseñanza de las ciencias.

Universidad Católica del Maule, juan.neira@alu.ucm.cl https://orcid.org/0000-0002-1332-9919 


\section{ABSTRACT}

The objective of this study was focused on conducting a review of the current state of experimental activities as a strategy to achieve scientific literacy at school stages. Currently, experimental activities, rather than being associated with problem-solving activities, are oriented to be practical complements of theoretical classes, which implies that it will cause a lack of interest in students for the study of science. Experimental activities are a valuable instance to bring students closer to the study of natural sciences, and we believe that maintaining the investigative character is essential to awaken interest and to achieve an adequate development of scientific literacy and, therefore, critical thinking.

Key words: Laboratory practical activity; scientific literacy; critical thinking; science teaching.

\section{INTRODUCCIÓN}

Uno de los pilares fundamentales para el desarrollo social y económico de cada país es la educación y particularmente el desarrollo del pensamiento crítico de sus ciudadanos. Diversos actores tales como políticos, científicos y docentes coinciden al indicar que la inversión y esfuerzo realizado por un gobierno en educar a la población es imperativa para convertirse en una nación desarrollada. Queda en evidencia, entonces, la importancia de la educación para el logro de una adecuada adquisición de conocimientos, habilidades y competencias para el desarrollo de la Alfabetización Científica y, por ende, del pensamiento crítico. Ahora bien, dentro de los niveles educativos, la educación media ha sido reconocida como una de las etapas fundamentales para establecer la Alfabetización Científica de los estudiantes (Vilches \& Gil, 2001), y el rol que desempeña el docente ha sido considerado trascendental para alcanzar dichos logros de aprendizajes del estudiantado, lo que sitúa al profesor como uno de los principales actores para el logro de un adecuado desarrollo de la Alfabetización Científica (Hattie, 2003).

Si observamos la situación de las ciencias naturales, una de las estrategias didácticas más recomendadas para el logro de la Alfabetización Científica son las Actividades Experimentales (AE) también Ilamadas actividad prácticas, que son instancias de construcción de conocimiento dadas sus características intrínsecas, tales como ser centradas en los estudiantes, implementar el método científico, utilizar materiales específicos, ser desarrollas en un ambiente diferente al aula tradicional y contribuir al 
desarrollo de competencias actitudinales, conceptuales y procedimentales (Espinosa et al., 2016). Existen, además de sus características intrínsecas, una serie de razones que fundamentan la importancia de las AE. Por ejemplo, ser determinante para el aprendizaje de los alumnos en la enseñanza de las ciencias (Lightburn \& Fraser, 2007), ser más efectivas para el logro de aprendizajes comparadas con otros métodos destinados a ese fin (Séré, 2002), permitir a los estudiantes mantener contacto directo con los fenómenos en estudio (Krasilchik, 2009), promover la construcción de modelos conceptuales en la enseñanza de la ciencia y ofrecer una visión correcta de la actividad científica (Fernández, 2013).

Sin embargo, y pese a las características que sugieren la importancia de la implementación de las $\mathrm{AE}$, se han identificado una serie de escenarios que dificultan la ejecución de éstas, tales como las nociones de los docentes vinculas a una representación tradicional de la ciencia, así como a una imagen instrumental, simple y demagógica sobre la enseñanza y el aprendizaje (Quintanilla et al., 2014), la escasa autonomía por parte de los docentes para la toma de decisiones (MacBeath, 2012), los escasos recursos y apoyo insuficientes, junto con un mal ambiente laboral (Klassen \& Chiu, 2010; MacBeath, 2012; Struyven \& Vanthournout, 2014), la realidad con que se enfrentan algunos docentes noveles durante sus primeras experiencias laborales (Silva et al., 2017), la sobrecarga laboral del profesorado (Barmby, 2006; Skaalvik \& Skaalvik, 2011 ), y el mal comportamiento de los estudiantes (Barmby, 2006).

Por tales razones, el objetivo del presente trabajo se centra en realizar una revisión y discusión del estado actual de la realización de las actividades experimentales como una estrategia para el logro de la alfabetización científica en etapas escolares.

\section{¿CÓMO SE EJECUTA LA EXPERIMENTACIÓN EN LA EDUCACIÓN BÁSICA Y MEDIA?}

Al analizar el estado actual de la realización de actividades prácticas, lo que observamos es que algunos estudios han dado cuenta que durante la ejecución de las AE, el estudiantado no aprende a cómo enfrentarse a la resolución de problemas científicos escolares, sino que a memorizar o reproducir fórmulas, definiciones, cálculos y procedimientos (Bodner \& Herron 2002), dejando poco o nulo espacio a otras actividades más desafiantes, como es el análisis de información mediante gráficos, tablas o la proposición de hipótesis frente a posibles soluciones de problemáticas de la cotidianidad escolar. Si observamos lo que sucede actualmente en Chile, vemos que la formación científica del alumnado tampoco parece ser muy prometedora. Por ejemplo, al analizar la enseñanza de las ciencias durante la educación media, observamos que ésta se 
realiza fundamentalmente a través de aspectos teóricos, caracterizados por un aprendizaje memorístico de contenidos desacoplados, con una comprensión de la ciencia más bien descontextualizada y alejada de lo cotidiano de los estudiantes (Albertini et al., 2005).

Si bien el estudio de Cofré et al., (2010), indicó que los estudiantes valoran positivamente las actividades prácticas, al mismo tiempo consideran que la forma en que se implementan, más que incentivar su curiosidad, tiende a que pierdan el interés por el estudio de las ciencias. Otro estudio encontró que durante el desarrollo de las AE, los estudiantes no emplean una adecuada metodología científica, y el énfasis de la enseñanza de las ciencias se centra más en los contenidos que en el desarrollo de habilidades y actitudes (Conzález et al., 2009), por lo que, en la mayoría de los casos, el trabajo de experimentación se dedica principalmente a la comprobación de conceptos que han sido previamente estudiados en clases teóricas (Cofré et al., 2010a; Fernandez, 2018; Zorrilla \& Mazzitelli, 2015). Pensamos que este es un aspecto crucial dentro de la educación de las ciencias, ya que si las AE que plantean los profesores no cautivan a sus estudiantes o bien, se utilizan únicamente con el objetivo de comprobar aspectos que han sido estudiados en las clases teóricas, posiblemente se desaprovecharán como estrategia didáctica y, fundamentalmente, como oportunidad para que los estudiantes despierten vocaciones por el estudio de las ciencias.

Tabla 1: Niveles de Abertura de Priestley.

\begin{tabular}{|c|c|c|c|}
\hline Nivel & Título & Descripción de las actividades en el laboratorio & $\begin{array}{c}\text { Proceso cognitivo } \\
\text { requerido }\end{array}$ \\
\hline 1 & $\begin{array}{c}\text { Herméticamente } \\
\text { cerrado }\end{array}$ & $\begin{array}{c}\text { Se proporcionan todos los procedimientos al alumnado. Los } \\
\text { estudiantes apuntan los datos en los huecos reservados de un } \\
\text { informe de laboratorio. Se incluyen tablas con los datos }\end{array}$ & Conocimiento \\
\hline 2 & Muy cerrado & $\begin{array}{c}\text { Se proporcionan todos los procedimientos a los estudiantes. Se } \\
\text { incluyen tablas de datos }\end{array}$ & Conocimiento \\
\hline 3 & Cerrado & Se proporcionan todos los procedimientos a los estudiantes & Conocimiento \\
\hline 4 & Entreabierto & $\begin{array}{c}\text { Se proporcionan todos los procedimientos a los estudiantes. } \\
\text { Algunas preguntas o conclusiones son abiertas }\end{array}$ & Comprensión y aplicación \\
\hline 5 & Ligeramente abierto & $\begin{array}{c}\text { Se proporcionan la mayoría de procedimientos a los estudiantes } \\
\text { y algunas preguntas o cuestiones son abiertas }\end{array}$ & Aplicación \\
\hline 6 & Abierto & $\begin{array}{c}\text { Los estudiantes desarrollan sus propios procedimientos. Se } \\
\text { les proporciona una lista con el material. Muchas preguntas o } \\
\text { conclusiones son abiertas }\end{array}$ & Análisis y síntesis \\
\hline
\end{tabular}

Fuente: Valverde et al. (2006). 
Asimismo, el estudio llevado a cabo por González-Weil et al. (2012), dio cuenta que la enseñanza de las ciencias se lleva a cabo principalmente mediante aspectos teóricos, lo que disminuye la capacidad de aprendizaje y motivación de los estudiantes (Guevara \& Lemus, 2019), y las AE propuestas por los textos más que estar asociada a procesos de indagación, están centradas en conocer y comunicar hechos científicos mediante tareas vinculadas a habilidades cognitivas de baja complejidad (Ruiz et al., 2016).

En este sentido, varios estudios coinciden en que las AE que se ejecutan con mayor frecuencia presentan bajos niveles de abertura (Valverde et al., 2006); es decir, observaciones y experimentos (Crisafulli \& Villalba, 2013), siendo las actividades basadas en la indagatoria (demostraciones, resolución de problemas y proyectos escolares) las que se realizan con menor periodicidad. Por ejemplo, el estudio llevado a cabo por Cofré et al. (2010), al consultar a docentes de enseñanza básica cuáles eran las AE que más utilizaban (figura 1), encontró que las observaciones y experimentos eran las más frecuentes, mientras que las actividades que involucran la creación de modelos y la resolución de problemas eran las que se llevaban a cabo con menor periodicidad. En este punto pensamos que, si bien todas las actividades son igualmente importantes, creemos que es relevante que los docentes se motiven no solamente a ejecutar actividades como observaciones de fenómenos científicos y experimentos para comprobar aspectos estudiados en clases teóricas, sino que también a implementar actividades que desafien al estudiante y le permitan desarrollar habilidades, competencias y aptitudes propias del área de las ciencias naturales.

Figura 1: Tipo de actividad de laboratorio más usada por profesores de enseñanza básica que hacen clases en el subsector de comprensión del Medio Natural.

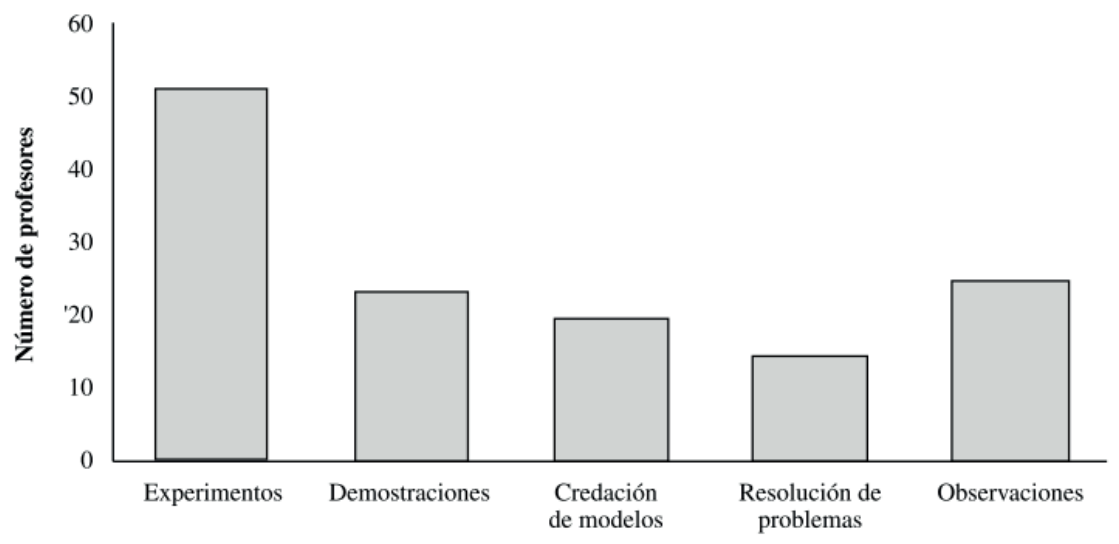

Tipo de actividad más usada

Fuente: Cofré et al. (2010). 


\section{LA IMPORTANCIA DE LA ACTIVIDAD EXPERIMENTAL}

Si bien es frecuente encontrar en la literatura que las AE a menudo son concebidas como una actividad complementaria a las clases teóricas, es bien sabido que la instancia práctica no debiera entenderse solamente como una actividad en que los estudiantes comprueban contenidos estudiados previamente en clases teóricas, sino que éstas debieran atenderse como un nexo para adquirir competencias y habilidades que permita al estudiantado desarrollar el pensamiento crítico. El estudio de González et al. (2009), considera a las AE una de las bases fundamentales de la indagación científica, en tanto permiten al estudiante construir su propio conocimiento, reflexionando acerca de qué sabe, y acerca de cómo lo ha llegado a saber, mejorando así su comprensión acerca de los procesos que llevan a la comunidad científica a generar conocimiento. Asimismo, un estudio previo de Cofré et al. (2015), indicó que la importancia de enfocar el estudio de las ciencias mediante la indagación científica, radica en que puede actuar como un ente catalizador que motive a los estudiantes a sentir interés por el mundo de las ciencias naturales. En este sentido, creemos que es fundamental canalizar los esfuerzos en dirección de poder aprovechar la experimentación como una instancia única para la enseñanza de las ciencias naturales, que al mismo tiempo puede ser empleada como una vía para despertar vocaciones e intereses en el estudiantado por el estudio de las ciencias.

Sin embargo, y aunque haya grandes ideas para reafirmar la importancia de las AE, el estudio llevado a cabo por Tellez et al. (2012), investigó las razones que motivan a los docentes a descartar este tipo de actividades. De este estudio, se encontró que las principales dificultades que se presentan al implementar las AE se vinculan a exigencias curriculares a las que se ven expuestos los profesores de aula, la falta de tiempo, de espacio y la carencia de insumos de laboratorio (equipos, instrumentos, reactivos, entre otros). Frente a la situación anterior, un estudio desarrollado por Carrascona et al. (2007), considera que sería sumamente relevante que se impulsaran medidas para la adquisición de insumos de trabajo según cuales fueran las características las comunidades educativas, siendo el profesorado el implicado en la realización de las actividades prácticas quien decidiera qué y cuándo adquirir. Siguiendo con el estudio de Carrascona et al. (2007), consideran, además, que sería esencial incorporar dentro de los planes de formación inicial docente la gestión del laboratorio escolar y todo lo relacionado con éste. Asimismo, el estudio de Carrascosa \& Domínguez, (2017), señaló que la formación inicial docente debería incluir temas que permitan profundizar más, sobre todo en aquellos conceptos básicos de las disciplinas que se habrán de enseñar. 


\section{¿CUÁLES SON LAS CARACTERÍSTICAS QUE DEBE POSEER LA ACTIVIDAD EXPERIMENTAL PARA EL LOGRO DE UNA ADECUADA ALFABETIZACIÓN CIENTÍFICA?}

Las AE constituyen una instancia única para el aprendizaje e internalización de habilidades y competencias propias de las ciencias, y representan una estrategia pedagógica privilegiada en tanto permiten el desarrollo de la Alfabetización Científica y pensamiento crítico. Ahora bien, existe una serie de competencias que son necesarias que los docentes de ciencias deben conocer al momento de ejecutar una actividad práctica para que den cuenta de una adecuada Alfabetización Científica en los educandos. Por ejemplo, Cofré et al. (2010) menciona que existen 6 características que una actividad práctica debe poseer para el correcto desarrollo de la Alfabetización Científica: primero, el conocimiento y aplicación de una didáctica efectiva de las ciencias; segundo, el dominio de la disciplina que enseñe el docente; tercero, el manejo del currículo y de diferentes metodologías de evaluación; cuarto, la generación de una relación de confianza y respeto con los alumnos; quinto, la capacidad de reflexión acerca de su práctica; y sexto, la permanente actualización y manejo de nuevas tecnologías. En este sentido, es importante destacar que la alfabetización científica y el desarrollo del pensamiento crítico no se logran por la implementación de las AE per se, sino que son necesarias algunas competencias y condiciones mínimas que deben cumplirse, lo cual nos hace pensar que tanto el perfeccionamiento como la constante reflexión del docente sobre su quehacer pedagógico son fundamentales para la consecución de dicho objetivo.

Un profesor que domina su disciplina, las estrategias metodológicas y, además, reflexiona sobre su práctica docente, será un profesor mucho más seguro, generando un clima de confianza entre sus estudiantes, por lo que podrá aplicar una didáctica de las ciencias de forma mucho más efectiva.

Siguiendo con esta línea, algunos autores sostienen que las AE que se ejecuten debieran constantemente presentar situaciones problematizadas, puesto que este tipo de actividades otorgan un mayor grado de autonomía al estudiantado (Quintanilla et al., 2010), y además permiten establecer una secuencia temporal constante de carácter indagatorio (Meroni et al., 2015). La importancia de problematizar las actividades radica en que son una competencia transcendental de pensamiento científico para los estudiantes de ciencias (Quintanilla et al., 2014), ya sea para ser profesionales o individuos competentes en el campo de la investigación, como también para ser ciudadanos responsables alfabetizados científicamente (Adúriz-Bravo et al., 2012). Frente a esta situación, resulta imperativo implementar actividades científicas que desafíen 
al estudiantado a enfrentar problemáticas de orden científico que les permita desarrollar habilidades y aptitudes, ya sea para que estén preparados para la consecución de estudios ligados a áreas científica como también para ser ciudadanos críticos y científicamente alfabetizados.

Creemos que una de las actividades importantes que se pueden realizar en etapas escolares son las ferias científicas, que han demostrado ser una herramienta valiosa para la adquisición de conocimientos y desarrollo de habilidades (Alvarado et al., 2018), que dada sus características intrínsecas, permiten a los estudiantes mantener el carácter indagatorio en forma constante. En este sentido, pensamos que acercar a los estudiantes a los laboratorios a través de la promoción de ferias científicas o proyectos de investigación escolar, es una forma efectiva para fortalecer la enseñanza-aprendizaje vinculada con las ciencias (Oppliger et al., 2019), además de ser un motor que les impulse a desarrollar vocaciones e intereses por el estudio de las ciencias. Por ejemplo, el estudio previo de Guevara \& Lemus, (2019) ha mostrado que en el caso particular de los estudiantes de educación media, participar en ferias científicas significa una posibilidad de experimentar una práctica positiva que los motiva a desarrollar su vocación científica, y que incide en sus decisiones de preferencia y elección de carreras relacionadas al momento de ingresar a la universidad.

Por otro lado, algunos estudios han mostrado que la formación académica del profesorado es otra arista fundamental para una correcta enseñanza de las ciencias. La formación inicial docente debe ya estar enfocada en el desarrollo de habilidades y competencias indagatorias, más que estar centradas en un proceso mecánico de transferencia de contenidos. Por ejemplo, el estudio de Cofré et al., (2010) indicó que la formación de los docentes de ciencias naturales debiera ser concomitante a los cambios en el currículo nacional de ciencias, el cual, a su vez, debiera tributar principalmente al desarrollo de habilidades de indagación más que a la adquisición de contenidos; y a las condiciones en que los docentes realizan sus prácticas de enseñanza de las ciencias, es decir, dedicar menos horas frente a los educandos, y más para reflexionar y planificar oportunidades de enseñanza-aprendizaje.

Por último, cabe destacar que el estudio de Bezanilla et al., (2018) al consultar a 230 académicos universitarios acerca de la ejecución de actividades prácticas durante la formación profesional de docentes, observaron que la mayoría la vinculan a procesos de análisis y razonamiento, solo algunos al cuestionamiento, evaluación y toma de decisiones, y muy pocos lo entienden como proceso de acción y compromiso. En este sentido, parece imprescindible que ya desde la formación profesional de docentes, 
se lleven a cabo actividades de indagación y reflexión de las prácticas, lo cual podría convertir la indagación científica como el principal enfoque pedagógico para el logro de una adecuada Alfabetización Científica, entendiendo este acto como un proceso reflexivo de exploración, planificación, comunicación, construcción y reconstrucción del quehacer docente (González-Weil et al., 2012).

\section{CONCLUSIONES}

Los datos que hemos presentado anteriormente muestran una panorámica general sobre el estado de Alfabetización Científica en la asignatura de ciencias naturales a través de la ejecución de las AE. La experimentación constituye una instancia única para el aprendizaje de las ciencias naturales, en tanto permiten al estudiantado establecer un contacto estrecho con la actividad científica, poniendo en práctica sus conocimientos, habilidades y competencias propias del área investigativa. Las AE son una estrategia valiosa de la que dispone el profesorado de ciencias, aunque se ha evidenciado que en la mayoría de los casos las actividades planteadas solo se emplean como un complemento para las clases teóricas, y en la mayoría de las situaciones las actividades son de observación y experimentación pauteada complemente por el profesor, mientras que las actividades de indagatoria como las demostraciones y resolución de problemas tienden a implementarse con menor frecuencia. Según la revisión de la literatura analizada, pensamos que es transcendental el mantenimiento del carácter indagatorio de las actividades para el establecimiento y desarrollo de una adecuada Alfabetización Científica en el estudiantado. La experimentación es una herramienta valiosa que puede ser una vía catalizadora para despertar vocaciones e intereses por parte de los estudiantes, y fomentar el uso e implementación de actividades problematizadas podría beneficiar en gran medida el desarrollo de competencias y habilidades científicas. Además de la implementación de actividades problematizadas, consideramos que es trascendental para el desarrollo de buenas prácticas escolares y desarrollo del pensamiento crítico en general, la presencia de un enfoque indagatorio desde la formación profesional del docente de ciencias, y también promover mejoras de las condiciones pedagógicas escolares bajo las cuales se enmarcan las actividades prácticas en la actualidad.

Finalmente, consideramos necesario remarcar la importancia de continuar investigando el rol e impacto que tiene el desarrollo de las competencias de Alfabetización Científica a través de la ejecución de la experiencia práctica basada en la indagatoria en los estudiantes en etapas escolares, ya que representan una instancia altamente 
fructífera para el desarrollo de habilidades, competencias y aptitudes, así como también de vocaciones e intereses por las ciencias. Por último, y a partir de la literatura revisada, hemos observado que, si bien se valora la práctica experimental, no hay mayores datos sobre las condiciones que determinan el logro de la implementación de las actividades experimentales y cómo algunas condiciones pueden afectar el desarrollo de las mismas. En este sentido, pensamos que futuros estudios deberían enfocarse en analizar algunas condiciones y/o variables que determinan la ejecución de las actividades prácticas, tales como el tipo de actividades que realizan (según el grado de indagatoria), la frecuencia con que se llevan a cabo, los recursos pedagógicos con que cuenta el profesorado, los espacios disponibles, los insumos de laboratorio que se emplean, cómo se evalúa el logro las actividades y las dificultades que se manifiestan durante la ejecución de las mismas, para poder canalizar esfuerzos en pos de una mejor práctica pedagógica y desarrollo de la alfabetización científica y pensamiento crítico en el estudiantado. 


\section{REFERENCIAS BIBLIOGRÁFICAS}

Adúriz-Bravo, A., Merino, C., \& Izquierdo, M. (2012). An approach to the construction of chemistry curricula on the basis of structuring theoretical fields | Una aproximación a la construcción del currículo de química sobre la base de la estructura de los campos teóricos. Journal of Science Education, 13, 42-44. Recuperado de: https://pure.pucv.cl/es/publications/an-approach-to-theconstruction-of-chemistry-curricula-on-the-bas

Alvarado, R., Armando, D., Vázquez, B., \& Camacho, M. (2018). Las Ferias de Ciencia y Tecnología de Costa Rica y sus aportes a la educación secundaria. Actualidades Investigativas En Educación, 18(2), 1-43. DOI: https://doi.org/10.15517/ aie.v18i2.33170

Barmby, P. (2006). Improving teacher recruitment and retention: The importance of workload and pupil behaviour. Educational Research, 48(3), 247-265. DOI: https://doi.org/1 0.1080/00131880600732314

Bezanilla, M. J., Poblete, M., Fernández, D., Arranz, S., \& Campo, L. (2018). El Pensamiento Crítico desde la Perspectiva de los Docentes Universitarios. Estudios Pedagógicos, 44(1), 89-113. DOI: https://doi.org/10.4067/s0718-07052018000100089

Carrascosa, J., \& Domínguez, C. (2017). Problemas que dificultan una mejor utilización de la Didáctica de las Ciencias en la formación del profesorado y en la enseñanza secundaria. Revista Científica, 3(30), 167-180. DOI: https://doi. org/10.14483/23448350.12289

Carrascosa, J., Payá, L., Domínguez, M. C., \& Climent, M. T. (2007). Sostenibilidad y laboratorios escolares. Educación Química, 18(4), 31 1. DOI: https://doi.org/10.22201/ fq. 18708404e.2007.4.65878

Cofré, H., Camacho, J., Galaz, A., Jiménez, J., Santibáñez, D., \& Vergara, C. (2010a). La educacion cientifica en Chile: Debilidades de la enseñanza y futuros desafios de la educacion de profesores de ciencia. Estudios Pedagogicos, 36(2), 279-293. DOI: https://doi.org/10.4067/s0718-07052010000200016

Cofré, H., Camacho, J., Galaz, A., Jiménez, J., Santibáñez, D., \& Vergara, C. (2010b). La educacion cientifica en Chile: Debilidades de la enseñanza y futuros desafios de la educacion de profesores de ciencia. Estudios Pedagogicos, 36(2), 279-293. DOI: https://doi.org/10.4067/s0718-07052010000200016 
Cofré, H., González-Weil, C., Vergara, C., Santibáñez, D., Ahumada, G., Furman, M., Podesta, M. E., Camacho, J., Gallego, R., \& Pérez, R. (2015). Science Teacher Education in South America: The Case of Argentina, Colombia and Chile. Journal of Science Teacher Education, 26(1), 45-63. DOI: https://doi.org/10.1007/s10972015-9420-9

Crisafulli, F., \& Villalba, H. (2013). Laboratorios para la enseñanza de las ciencias naturales en la educación media general. Educere: Revista Venezolana de Educación, 17(58), 475-485. Recuperado de: https://www.redalyc.org/ pdf/356/35630404011.pdf

Espinosa, E., González, K., \& Hernández, L. (2016). Las prácticas de laboratorio: una estrategia didáctica en la construcción de conocimiento científico escolar. Entramado, 12(1), 266-281. DOI: https://doi.org/10.18041/entramado.2016v12n1.23125

Fernandez, N. (2018). Actividades prácticas de laboratorio e indagación en el aula. Tecné, Episteme y Didaxis: TED, 44, 203-218. Recuperado de: http://www.scielo. org.co/pdf/ted/n44/0121-3814-ted-44-203.pdf

Fernández, N. (2013). Los Trabajos Prácticos de Laboratorio por investigación en la enseñanza de la Biología. Revista de Educación En Biología, 16(2), 15-30. Recuperado de: https://revistas.unc.edu.ar/index.php/revistaadbia/article/view/22395

González-Weil, C., Cortéz, M., Bravo, P., Ibaceta, Y., Cuevas, K., Quiñones, P., Maturana, J., \& Abarca, A. (2012). La indagación científica como enfoque pedagógico: Estudio sobre las prácticas innovadoras de docentes de ciencia en EM (Región de Valparaíso). Estudios Pedagogicos, 38(2), 85-102. DOI: https://doi. org/10.4067/S0718-07052012000200006

González, C., Martínez, M. T., Martínez, C., Cuevas, K., \& Muñoz, L. (2009). La educación científica como apoyo a la movilidad social: desafíos en torno al rol del profesor secundario en la implementación de la indagación científica como enfoque pedagógico. Estudios Pedagógicos (Valdivia), 35(1), 63-78. DOI: https:// doi.org/1 0.4067/S0718-07052009000100004

Guevara, M., \& Lemus, M. (2019). Las jornadas científicas fortalecen la enseñanza-aprendizaje de aspectos biológicos en educación primaria y secundaria. Revista Caribeña de Investigación Educativa (RECIE), 3(1), 95-101. DOI: https://doi. org/10.32541/recie.2019.v3i1.pp95-101 
Hattie, J. (2003). Teachers make a difference. In Paper presented at the Australian Council for Educational Research Annual Conference on Building Teacher Quality. DOI: https://doi.org/10.1080/0300443920830113

Klassen, R. M., \& Chiu, M. M. (2010). Effects on Teachers' Self-Efficacy and Job Satisfaction: Teacher Gender, Years of Experience, and Job Stress. Journal of Educational Psychology, 102(3), 741-756. DOI: https://doi.org/10.1037/a0019237

Krasilchik, M. (2009). Introdução à didàtica da biologia. In Introdução à Didática da Biologia. Escrituras Editora.

Lightburn, M. E., \& Fraser, B. J. (2007). Classroom environment and student outcomes among students using anthropometry activities in high-school science. Research in Science and Technological Education, 25(2), 153-166. DOI: https:// doi.org/10.1080/02635140701250576

MacBeath, J. (2012). The future of the teaching profession. In Management in Education. Cambridge: Educational International Research Institute and University of Cambridge.

Meroni, G., Copello, M. I., \& Paredes, J. (2015). Enseñar química en contexto. Una dimensión de la innovación didáctica en educación secundaria. Educacion Quimica, 26(4), 275-280. DOI: https://doi.org/10.1016/j.eq.2015.07.002

Oppliger, L. V., Nuñez, P., \& Gelcich, S. (2019). Ferias Científicas como Escenarios de Motivación e Interés por la Ciencia en Estudiantes Chilenos de Educación Media de la Región Metropolitana. Información Tecnológica, 30(6), 289-300. DOI: https://doi.org/1 0.4067/S0718-07642019000600289

Quintanilla, M., Joglar, C., Jara, R., Camacho, J., Ravanal, E., Labarrere, A., Cuellar, L., Izquierdo, M., \& Chamizo, J. A. (2010). Resolución de problemas científicos escolares y promoción de competencias de pensamiento científico. ¿Qué piensan los docentes de química en ejercicio? Enseñar Ciencias En El Nuevo Milenio. Retos y Desafíos, 28(2), 185-198. Recuperado de: http://dialnet.unirioja.es/servlet/articulo?codigo=3317405

Quintanilla, M., Joglar, C., Labarrere, A., Merino, C., Cuellar, L., \& Koponen, I. (2014). ¿Qué piensan los profesores de química en ejercicio acerca de la resolución de problemas científicos escolares y sobre las competencias de pensamiento cien- 
tífico? Estudios Pedagogicos, 40(2), 265-284. DOI: https://doi.org/10.4067/ s0718-07052014000300017

Ruiz, M., Montenegro, M., Meneses, A., \& Venegas, A. (2016). Oportunidades para aprender ciencias en el currículo chileno: Contenidos y habilidades en educación primaria. Perfiles Educativos, 38(153), 16-33. DOI: https://doi.org/10.22201/ iisue.24486167e.2016.153.57633

Séré, M.-G. (2002). La enseñanza en el laboratorio. ¿Qué podemos aprender en términos de conocimiento práctico y de actitudes hacia la ciencia? Enseñanza de Las Ciencias: Revista de Investigación y Experiencias Didácticas, 20(3), 357-368. Recuperado de: https://www.raco.cat/index.php/Ensenanza/article/ view/21824

Silva, A. G., Navarrete, M. C., Conejeros, F. P., \& Devia, D. M. (2017). Abandono de la profesión docente en Chile: Motivos para irse del aula y condiciones para volver. Estudios Pedagogicos, 43(1), 123-138. DOI: https://doi.org/10.4067/S071807052017000100008

Skaalvik, E. M., \& Skaalvik, S. (2011). Teacher job satisfaction and motivation to leave the teaching profession: Relations with school context, feeling of belonging, and emotional exhaustion. Teaching and Teacher Education, 27(6), 1029-1038. DOI: https://doi.org/10.1016/j.tate.2011.04.001

Struyven, K., \& Vanthournout, G. (2014). Teachers' exit decisions: An investigation into the reasons why newly qualified teachers fail to enter the teaching profession or why those who do enter do not continue teaching. Teaching and Teacher Education, 43, 37-45. DOI: https://doi.org/10.1016/j.tate.2014.06.002

Tellez, M., Barragán, D., \& Casas, J. (2012). Transformación De Prácticas De Enseñanza A Nivel Universitario En El Laboratorio: Un Estudio De Caso Desde Los Niveles De Abertura. Recuperado de: https://www.researchgate.net/publication/270162254_Transformacion_De_Practicas_De_Ensenanza_A_Nivel_Universitario_En_El_Laboratorio_Un_Estudio_De_Caso_Desde_Los_Niveles_De_ Abertura

Valverde, J., Jiménez, L., \& Viza, L. (2006). La atención a la diversidad en las prácticas de laboratorio de química : los niveles de abertura. Enseñanza de Las Ciencias: Revista de Investigación y Experiencias Didácticas, 24(1), 59-70. Recuperado de: https://www.raco.cat/index.php/Ensenanza/article/view/73532 
Vilches, A., \& Gil, D. (2001). Una alfabetización científica para el siglo XXI: Obstáculos y propuestas de actuación. In Investigación en la escuela (Issue 43, pp. 27-37).

Zorrilla, E., \& Mazzitelli, C. (2015). Una propuesta de trabajos prácticos de laboratorio en física para el ingreso en carreras de formación docente. Revista de Enseñanza de La Física, 27(1), 703-707. Recuperado de: www.revistas.unc.edu.ar/ index.php/revistaEF/ 